\title{
Meso-design of heterogeneous dielectric material systems: Structure property relationships
}

\author{
Jeffrey Baker*, Jon Michael Adkins, Fazle Rabbi, Qianlong Liu, \\ Kenneth Reifsnider and Rassel Raihan \\ Department of Mechanical Engineering \\ University of South Carolina \\ Columbia, South Carolina 29208, USA \\ *Bakerjm8@cec.sc.edu
}

Received 17 October 2013; Revised 30 January 2014; Accepted 13 March 2014; Published 7 May 2014

\begin{abstract}
Heterogeneous materials are inherently dielectric, and charge distribution and transport in such materials involves complex local fields and polarizations that are remarkably sensitive to morphology and the interaction of conduction and permittivity. Trial and error design of such material systems is time consuming and expensive, and often ineffectual. However, heterogeneous materials are essential for energy conversion and storage, and they have become the foundation for major advances in the performance of devices such as batteries, fuel cells, separation membranes, and solar cells. The present paper presents some relationships in support of rational design based on an extensive experimental validation of the concepts and analysis that form a foundation for that design. Salient results include the prediction and confirmation of volume fraction effects (including nondilute mixtures), and the prediction and direct measurement of surface charge effects at internal interfaces as a function of constituent morphology and orientation.
\end{abstract}

Keywords: Heterogeneous material systems; dielectric design; surface charge.

\section{Introduction and Background}

Heterogeneous materials are, essentially, universally dielectric. The opportunity to create anomalously large dielectric responses in carefully structured composite materials for energy storage and conditioning and to mitigate losses associated with internal polarization for many common manmade electronic devices by the design of heterogeneous nano or microstructure has long been recognized. ${ }^{1}$ This paper provides elements of the basic foundation required for a design approach for such material systems. The physics of the dielectric response of heterogeneous materials is widely explored and discussed, and is not the focus of the present work. None the less, to the best of our knowledge, the scientific literature lacks a general approach to the design of heterogeneous material systems for this purpose.

The framework for our present discussion is heterogeneous functional material, i.e., heterogeneous material systems which actively interact with and often alter or transform the fields applied to them. Examples are found in devices such as the electrodes and membranes that are used to make fuel cells, electrolyzers, batteries, and separation membranes. A host of natural materials and organisms constitute examples of heterogeneous material systems. These include the lungs of animals, living bone, wood, and the cornea of the eye of mammals. We will refer to such material systems as HeteroFoaMs. HeteroFoaM materials are typically functional mixtures of two or more constituents and often a third void phase. ${ }^{2}$ An example is shown in Fig. 1.
Recent advances in such materials include the adaption of coherent boundary design methods and the creation of anomalous "giant" and "colossal" response in nanostructured materials. ${ }^{3,4}$ In many heterogeneous materials, particle boundary, shape, and size effects are evident. For disordered boundaries this is especially dominant. As an example, in radar absorbing materials very specific secondphase shapes, sizes, and arrangements are key to the design of the dielectric. ${ }^{5}$

The emergence of anomalous extreme values of global permittivity is associated only with the interactions of local fields created by charge storage at the boundaries of the constituent particles. Sample preparation in our laboratory has produced epoxy samples with embedded $40 \mu \mathrm{m}$ nominal size graphite flakes. The nondilute mixture contained in these samples shows anomalous emergent properties that are not bounded by the intrinsic constituent values. Figure 2 below shows the resulting Broadband Dielectric Spectroscopy $(\mathrm{BdDS})^{6}$ measurements for the epoxy matrix graphite flake samples. The real part of the conductivity of the $30 \%$ mixture was of the order of $10^{-7} \mathrm{~S} / \mathrm{cm}$ which is well below a conduction threshold. $^{7}$

The heterogeneity of a material system is a useful design parameter. Simply stated there are no simple conduction paths in HeteroFoaMs. The problem of conduction in composite materials as a function of microstructure has been widely discussed in the literature. ${ }^{8-13}$ However, the question of how to design the morphology of composite materials, especially with disordered boundaries, to achieve specific

This is an Open Access article published by World Scientific Publishing Company. It is distributed under the terms of the Creative Commons Attribution 3.0 (CC-BY) License. Further distribution of this work is permitted, provided the original work is properly cited. 


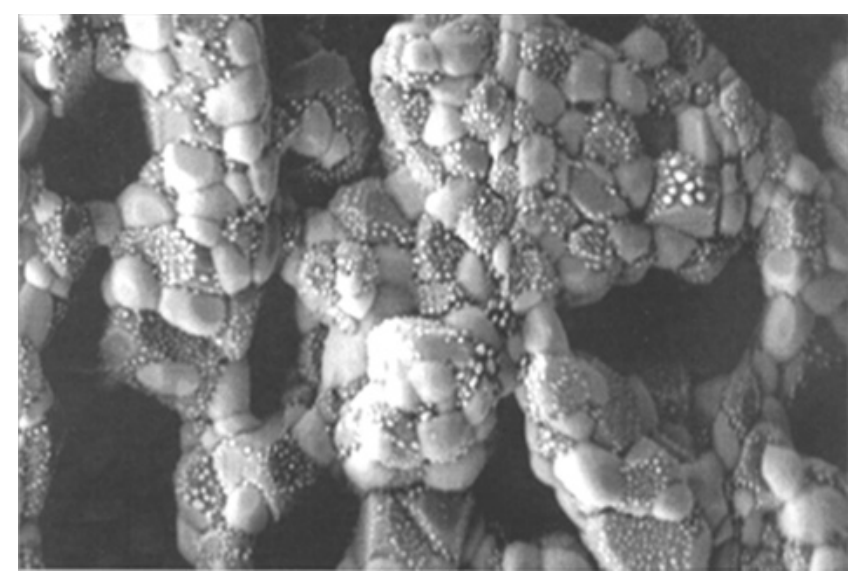

Fig. 1. Example of heterogeneous functional material morphology (www.HeteroFoaM.com).

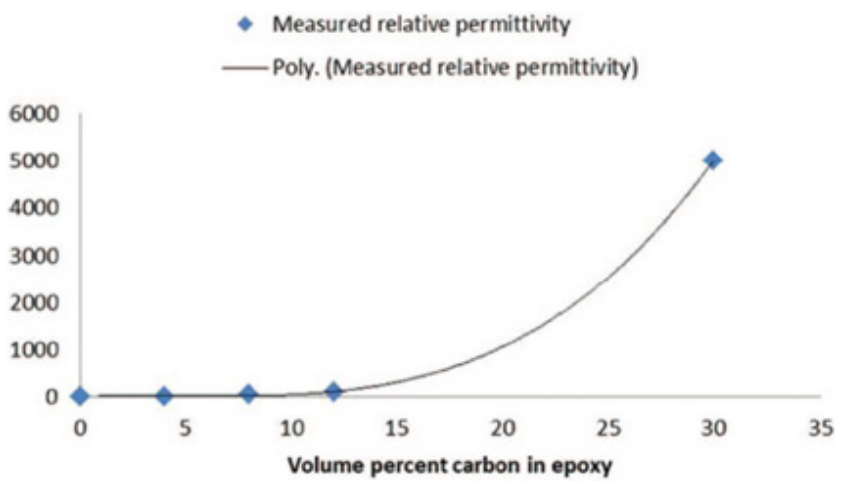

Fig. 2. Measured composite permittivity of carbon flakes embedded in an epoxy matrix for increasing volume fraction.

dielectric properties, large or small, has not been widely addressed. This is essential science and technology not only for the creation of energy storage devices where, for example, high capacitance is desirable but also for the development of fuel cells and a variety of electronic devices wherein internal dielectric polarization must be minimized and mitigated. An example can be seen in the control of pulse-power inputs such as lightning strikes in composite structures.

The present paper addresses the problem of constructing a foundation for the design of heterogeneous materials and material systems for specific dielectric response at the global level based on the properties, sizes, shapes, and interfaces of the constituents at the meso-level. Principle conformal analysis is translated into design concepts, which are extensively validated with first-ever data from experiments at the mesolevel to confirm the concepts and methods.

\section{Principle Concepts}

In general, when heterogeneous dielectric materials are immersed in an electric field, electric charges accumulate at the discontinuity created by the interface between the different phases. ${ }^{14}$ This effect is typically called Maxwell-WagnerSillars polarization and is an interfacial polarization at the mesocopic scale. ${ }^{15,16}$ The physics of this situation can be described by the use the usual linear concept of currents, $J$ and charge displacements, $D$ in the presence of a vector electric field, $E$ which may vary with time, and we state that

$$
J=\sigma \cdot E, \quad D=\varepsilon \cdot \varepsilon_{o} \cdot E,
$$

where $\sigma$ is the conductivity of a given material phase, $\varepsilon$ is the relative permittivity of that phase and $\varepsilon_{o}$ is the permittivity of vacuum. Then we invoke conservation of charge and the appropriate Maxwell's equation (in the absence of magnetic effects) to write

$$
\nabla J+\frac{d}{d t}(\Delta D)=0, \quad E=-\Delta \phi^{\prime}
$$

where $\phi^{\prime}$ is the local point-wise potential value in the material. Then combining we obtain the equation that controls the transient response as

$$
\nabla\left(\sigma \cdot \nabla \phi^{\prime}\right)+\frac{d}{d t} \nabla\left(\varepsilon \cdot \varepsilon_{\mathbf{o}} \nabla \phi^{\prime}\right)=0 .
$$

For steady state sinusoidal situations in which we can represent the potential as being the result of a harmonic input as

$$
\phi^{\prime}=\phi \cdot \mathrm{e}^{i w t}
$$

we find another useful form of Eq. (3)

$$
\nabla\left[\left(\sigma+i \omega \varepsilon \cdot \varepsilon_{0}\right) \cdot \nabla \phi\right]=0,
$$

where $\omega$ is the frequency of the input signal. Equation (5) is dispersive with frequency-dependent inputs and reduces to a conduction problem when $\omega=0$. For the present discussion, we will use Eqs. (3) and (5) as the foundation for our design concept. At the general level, Eq. (5) teaches us that in a heterogeneous material the product of the physical properties (some form of the conductivity and permittivity) and the slope of the potential must be a constant as we cross material boundaries. For the quasi-static case with harmonic inputs fields, the gradient of that product vanishes. ${ }^{17}$

The interacting field is a result of the charge in the electric field at the interface, and unless the conductivity and permittivity of adjacent material phases are identical, there is a disruption of charge transfer at the material boundary which results in internal polarization.

We note that the focus of the current work does not include the effect of experimental details at the boundaries. The boundary conditions are taken to be simply the voltages applied by contact plates. Rather, in regions away from the boundaries the equations are shown to hold. Our analysis is based on a two-dimensional solution, the results of which are compared to measurements on the free face of an axisymmetric specimen with uniform applied conditions along the $z$-axis. ${ }^{18}$

To illustrate this concept, we consider an electric field across a 2D representative volume element, depicted in Fig. 3 below where $\varepsilon$ is the dielectric constant of the material. 


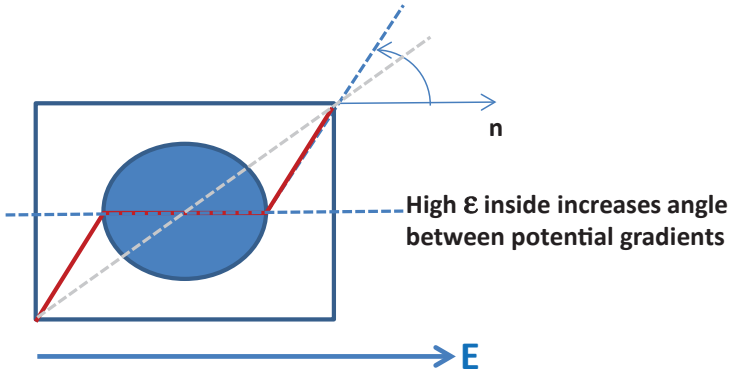

(a)

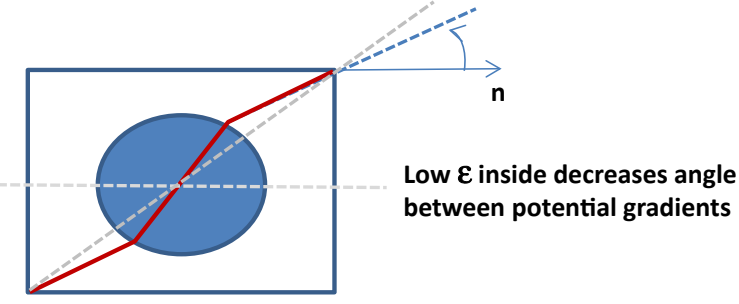

(b)

Fig. 3. (Color online) Graphical interpretation of Eq. 5. (a) Sample material with high $\varepsilon$ material as the included phase. (b) Material system with low $\varepsilon$ material as the included phase.

We observe that the charge distribution at the local level is disturbed by the electric field of the second phase material. In the presence of an electric field, the potential in a homogeneous material would have a constant slope as suggested by the diagonal dotted line in Figs. 3(a) and 3(b). If a second phase is added to create a composite element, and if the second phase has either higher conductivity or larger dielectric charge displacements, the slope of the potential is altered as shown by the red line in the figures. The constant product term in (5) implies that the field adjusts inside the material. Recalling basic electromagnetic theory, Gauss's law tells us that the change in the slope of the potential across a boundary is proportional to the surface charge, so when a more "conductive" material is inserted in our control volume we predict that the global capacitance or permittivity is increased, in proportion to the changes in slope shown in Fig. 3(a).

If a low permittivity/low conduction second phase is added to our control volume (lower than the surrounding matrix), then our logic predicts that the measured value of the global permittivity or capacitance should decrease, proportionally, as indicated in Fig. 3(b). The same framework of this model can be applied to multiphase material systems, e.g., to a crack inside of a composite material where the fiber, matrix and air void constitute the three separate distinct phases, or for coated particles, etc.

\subsection{Predicted permittivity}

In earlier work, we have developed very precise solutions to Eqs. (3) and (5), some in closed form for nondilute mixtures in which the local fields of the included particles interact across the material boundaries. ${ }^{7,19-21}$ Only a short summary of that work is given here. Further information on mixtures with dispersed particles can be obtained from existing scientific works. ${ }^{22,23}$

We set the present problem with a square material domain with an inclusion, weakly dielectric and relatively conductive, located at the material center embedded in a weakly conductive and strongly dielectric matrix surrounding it. The left and right boundaries of the material domain consisted of two fixed plate electrodes that provided an $\mathrm{AC}$ voltage difference, which was used to calculate the capacitance of the composite material assembly.

The top and bottom of the domain were periodic boundaries free of applied charge. In order to accurately predict the capacitance, it is crucial to satisfy the discontinuous interfacial conditions at the interface between two different materials and to accurately account for the interactions between particles, fields, and boundaries, a capability that has been extensively validated for our algorithm. ${ }^{19-21}$ The relative static permittivity of the matrix was set to 5 and its $\epsilon^{\prime \prime}$ value is set to 0.01 . Any small number compared to 5 is valid for the results reported here. For $\omega=10 \mathrm{~Hz}$, the corresponding conductivity was $8.854 \times 10^{-13} / \mathrm{m}$, i.e., we considered a weakly conductive matrix. The relative static permittivity of the inclusion was set to unity. This was relatively small compared to that of the matrix, and its real permittivity value was set to $10^{-3}, 6.5,100$, and $10^{5}$, which correspond to, for $\omega=10 \mathrm{~Hz}$, conductivities of $8.854 \times 10^{-14} \mathrm{~S} / \mathrm{m}, 5.755 \times$ $10^{-10} \mathrm{~S} / \mathrm{m}, 8.854 \times 10^{-9} \mathrm{~S} / \mathrm{m}$, and $8.854 \times 10^{-6} \mathrm{~S} / \mathrm{m}$. Although the conductivities of the inclusions were low, their consequences can be quite pronounced.

Figure 4(a) shows the effect of the extrinsic properties on the effective static permittivity of the material for different inclusions with the same intrinsic properties. For the inclusion in this case, we have set the real permittivity to 100 .

The effects of the shape and orientation of the inclusions is pronounced. Just changing the orientation of the elliptic inclusion to coincide with the horizontal direction of the applied field significantly increased the effective static permittivity. For example, for the volume fraction 0.5 , the effective static permittivity for vertical and horizontal inclusions reaches values of about 12.5 and 22.5, respectively. Solely by changing the orientation of the elliptic inclusion resulted in, the effective static permittivity increasing by about $80 \%$, a general result not previously reported to our knowledge.

The distance between this interfacial charge region and the electrode on the domain surface for the vertical inclusion is obviously further than it is for the horizontal inclusion orientation. Knowing the electrical field was oriented in the horizontal direction, and given the basic laws of electrostatics, 


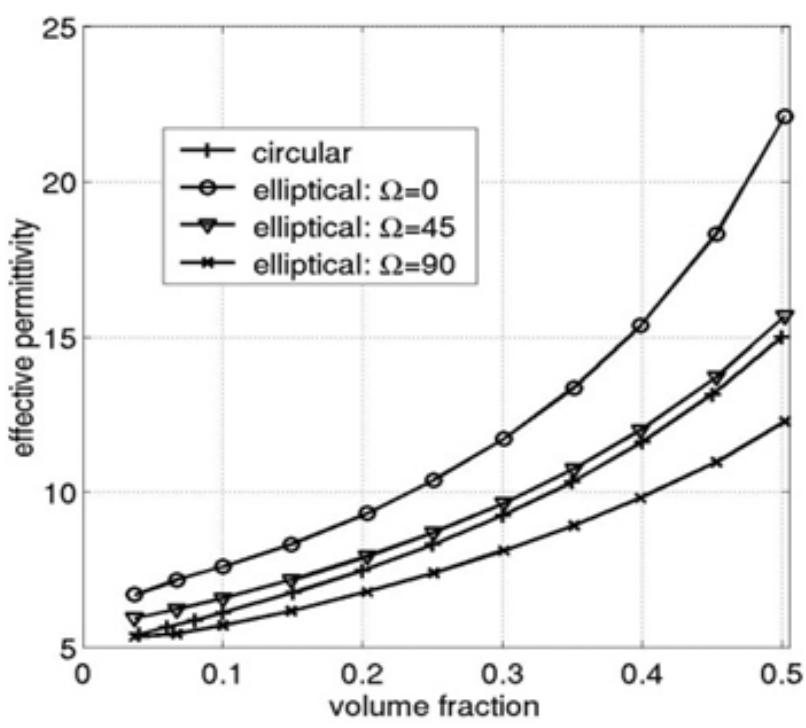

(a)

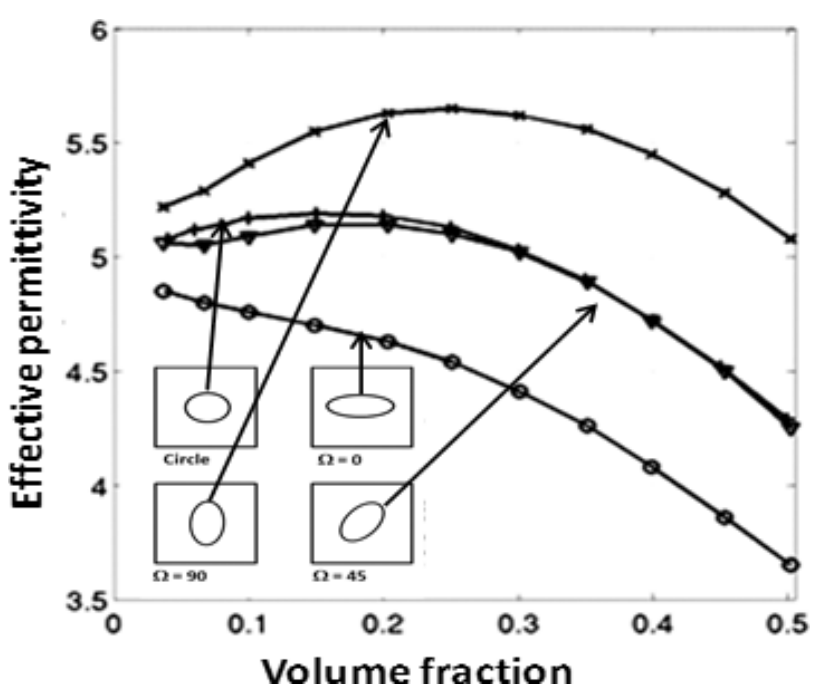

(b)

Fig. 4. Predicted variation of global effective permittivity with volume fraction of inclusions with higher conductivity than the matrix, (a), and for inclusions with somewhat lower permittivity than the matrix, (b).

we conceptually know that the interfacial charge will accumulate at the region closest to the electrodes.

This in turn supports the simple parallel plate capacitor concept that the capacitance is inversely related to the distance between the opposing charges, resulting in high effective static permittivity for the horizontal inclusion. However, the present work shows that not only the scalar distance, but the size, shape and orientation of the constituent phases as well as their relative conductive and dielectric properties all interact to determine the resulting capacitive character of the heterogeneous material.

Figure 4(b) shows the effect of the extrinsic properties on the effective static permittivity of the material for different inclusions with the same intrinsic properties. For the inclusion, we use a permittivity of 6.5 . The results show several remarkable features. First, the effects of the shape and orientation of the inclusions for the same volume fraction are pronounced. The horizontal and vertical arrangements of the inclusions give two extreme results while the slanted orientation with $\Omega=45$ gives results close to those of a circular particle, both of them between the two extreme results. Second, the effective permittivity is strongly nonlinearly dependent on the volume fraction for relatively large volume fractions while it is essentially linear for small volume fractions.

For nondilute heterogeneous materials, the strong interactions between particles, fields, and boundaries are the source of the nonlinear dependence on volume fraction, while for dilute material mixtures, the interactions are sufficiently weak resulting in linear dependence. This feature shows that our algorithm captures the material-field interactions while it exactly satisfies the discontinuous interface conditions. Third, as the size of the inclusions increases, the effective static permittivity does not necessarily increase monotonically.
For example, for the vertical inclusion, as the volume fraction increases, the effective static permittivity first linearly increases, reaches a peak value, and then decreases. This surprising result is due to the combined effects of the relatively small permittivity and relatively small but finite conductivity of the inclusion. More generally, for small volume fractions the creation of interfacial surfaces increases charge storage, whereas for larger volumes the permittivity of the inclusion volume compared to the matrix (smaller in our case) will dominate the changes.

In order to create extreme values of effective permittivity, these intrinsic and extrinsic aspects of heterogeneous materials should be designed so that the negative and positive charge regions are as close as possible. This is analogous to an electrical double layer with very small thickness between positive and negative layers of charge accumulation. In this context, heterogeneity is the key to enhanced charge and energy storage.

Based on our discussion of Fig. 3 and the concept of interpreting Eqs. (3) and (5) in terms of slopes of potential, the general features seen in Fig. 4 are expected; included phases with greater conductivity or permittivity should increase global capacitance (Fig. 4(a)), and if the included phase has a lower combination of conductivity and capacitance the global value should drop for large volume fractions Fig. 4(b). We also see an interesting increase before the expected decrease in Fig. 4(b), a feature that we will use to test our understanding of the physical behavior. This behavior is directly caused by the buildup of charge at interfaces. Initially, the surface area dominates the measured permittivity but the volume effects quickly emerge and dominate the overall value of the dielectric constant.

The simulation convention is that the $0^{\circ}$ orientation has the major axis of an elliptical inclusion parallel to the electric 
field and likewise perpendicular for the $90^{\circ}$ case. As seen in Fig. 4, the predicted permittivity at each volume fraction is highest at $0^{\circ}$ and lowest at $90^{\circ}$ while the $45^{\circ}$ orientation values are intermediate.

\section{Measured Values}

By constructing samples as noted above and making Broadband Dielectric Spectroscopy (BbDS) measurements on the free face perpendicular to the $z$-axis, the values illustrated in Fig. 4(a) were experimentally verified for the $0^{\circ}$ and $90^{\circ}$ orientations. By varying the ellipse dimensions the percent volume fraction of the inclusion was systematically controlled.

A three part cubic mold was designed, and ultimately yielded cubic samples with a dimension of $14.5 \mathrm{~mm}$ per side. The inclusions were made out of a low temperature tin solder. A two part mold was designed and manufactured in-house. Melted tin was poured in this mold resulting in inclusions that represented volume fractions of $10 \%, 20 \%$, and $30 \%$. Additionally, machined aluminum inserts enabled the inclusion to be carefully centered at orientations of $0^{\circ}, 45^{\circ}$ and $90^{\circ}$, see Fig. 5 below.

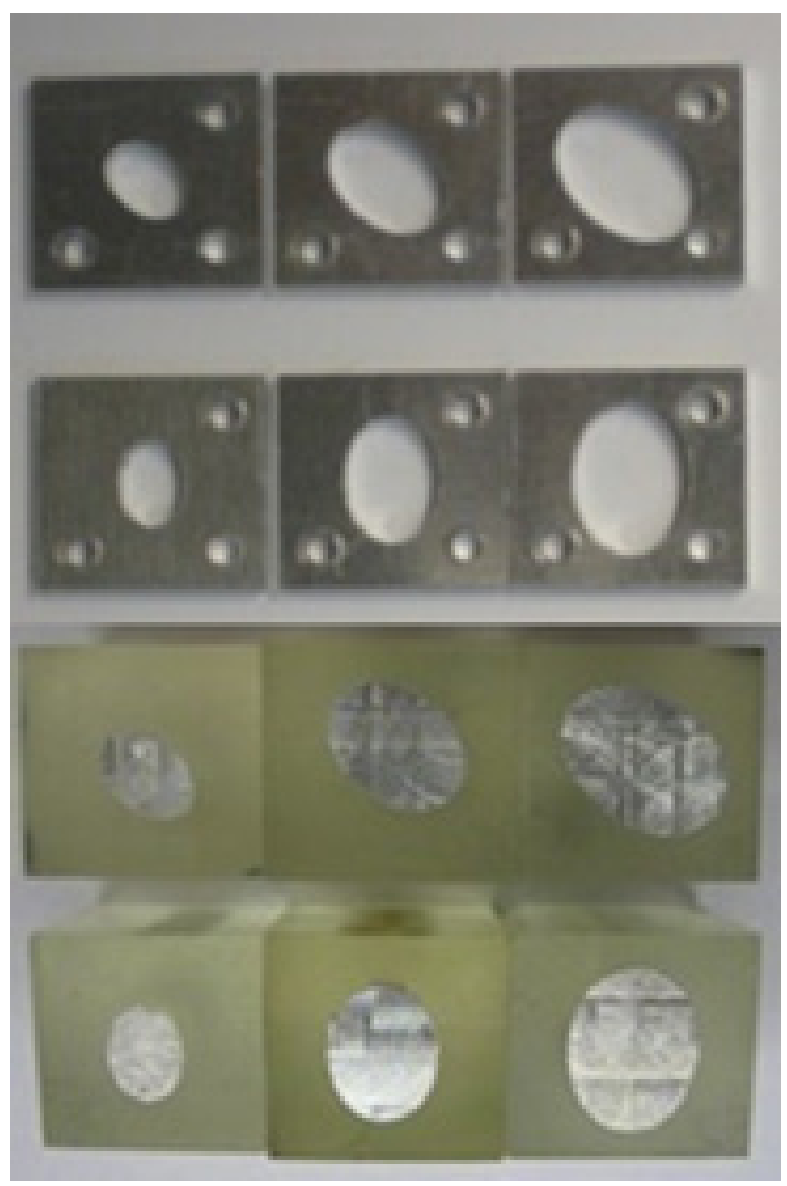

Fig. 5. Fabricated inclusion inserts (top). Completed tin epoxy samples (bottom).
The metal inserts were specifically designed for a tight fit with the tin inclusion. This ensured that each inclusion was parallel to the vertical direction. Likewise, the specific dimensions of the inserts were held in close tolerance with the dimensions of the base of the three part mold, which ensured repeatability in centering the inclusion in the middle of the mold base plate.

After the epoxy pour and cure, each sample was milled and polished to accommodate the dimensions of the BbDS electrode clamp. Figure 5 shows completed samples with variations in the inclusion volume fraction and orientation angle.

The BdDS clamp consisted of two round polycarbonate disks with milled grooves and two threaded screw holes. Each disk accommodated a square copper electrode with a length of $12 \mathrm{~mm}$ per side and a center groove to ensure proper spacing for the soldered electrical connection. Both electrodes were mounted on a compliant substrate in their respective halves. Two set screws and corresponding springs were used to join the polycarbonate halves and ensure uniform pressure between the two electrodes.

Three different samples with $10 \%$ volume fraction positioned at the $0^{\circ}$ orientation in the test fixture were measured by BbDS methods described in previous work. ${ }^{6}$ The measured real permittivity values at $10.76 \mathrm{~Hz}$ corresponded well with the $10 \mathrm{~Hz}$ frequency used in the predictions. These values were pulled from the frequency sweep data sets. For each sample, 12 individual measurements were made. Between each measurement the sample was removed then reseated in the test clamp, thereby minimizing the effect of electrode pressure variations across the sample surface. A total of 36 different measured values were averaged together and this value was plotted against the volume fraction of the inclusion. Similarly, this procedure was repeated for the $20 \%$ and $30 \%$ volume fractions.

The results are shown as Fig. 6 below. The black triangles in the plots indicate maximum, average, and minimum measured values for each volume fraction. Fig. 6 shows that

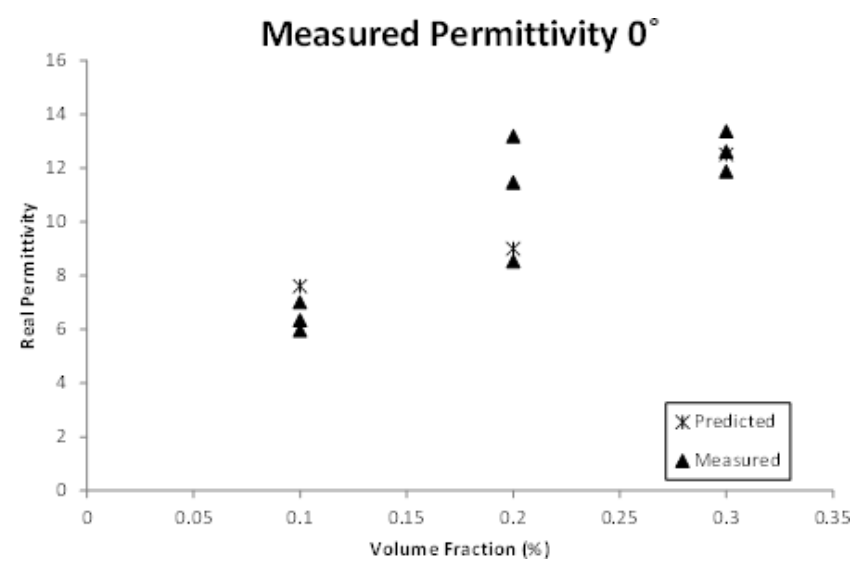

Fig. 6. Comparison of predicted and measured real permittivity values at $0^{\circ}$ orientation. 


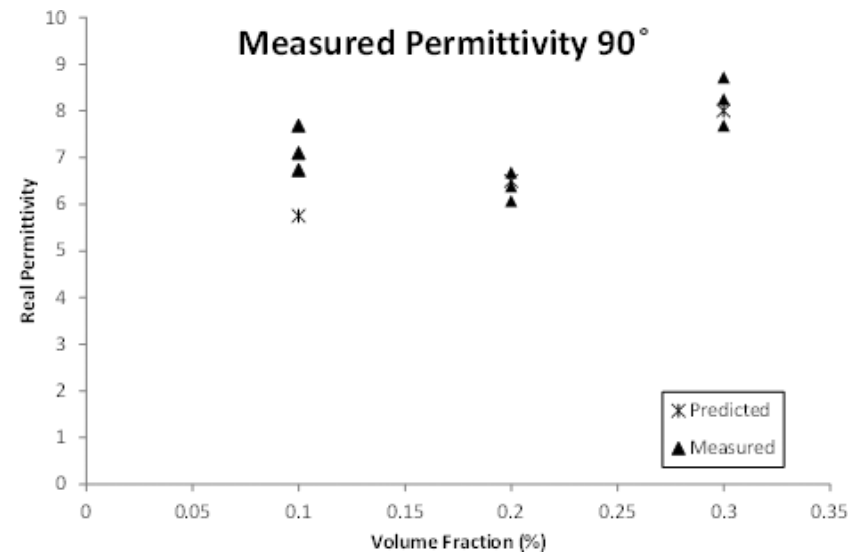

Fig. 7. Comparison of predicted and measured real permittivity values at $90^{\circ}$.

the predicted values, for the $0^{\circ}$ orientation shown in Fig. 4(a), fall almost completely within the experimental error of the measured permittivity values.

Next, following the same experimental procedure mentioned above, the sample's real permittivities at $10.76 \mathrm{~Hz}$ were measured at the $90^{\circ}$ orientation. Again, the resulting plot in Fig. 7 shows that the predicted values for the $20 \%$ and $30 \%$ volume fractions fall within the experimental error of the measured values.

As seen in both Figs. 6 and 7 above there is some variability between the predicted and measured values. However, by taking into account the slight imperfections in the sample fabrication process these differences can be resolved. For instance, the discrepancy at the $10 \%$ volume fraction seen above in Fig. 7 was removed on the $90^{\circ}$ face in contact with the electrode. This resulted in uneven contact between the sample and the test electrodes.

In both orientations, as the volume fraction increases, so does the value of the measured real permittivity. From a conceptual standpoint this makes sense. By design, there is a fixed volume for each sample. The effective permittivity of the metal is much greater than the relative permittivity value of epoxy, which is typically found to be around 4. Any increase in the volume of the tin inclusion is expected to increase the overall permittivity of the sample volume.

\section{Validation and Direct Measurement of Charge Distribution in Heterogeneous Materials}

To validate our predictions seen in Fig. 4(a) let us consider, for example the problem depicted in Fig. 8 which shows a simple cylindrically shaped high-dielectric phase in a low dielectric medium. Figure 8 shows the basic sample configuration consisting of a conductive inclusion and dielectric matrix. The slope is constant in the epoxy matrix on both sides of the inclusion, which is expected due to the uniformity of the epoxy throughout the sample volume. Likewise the near zero change in voltage across the conductive inclusion

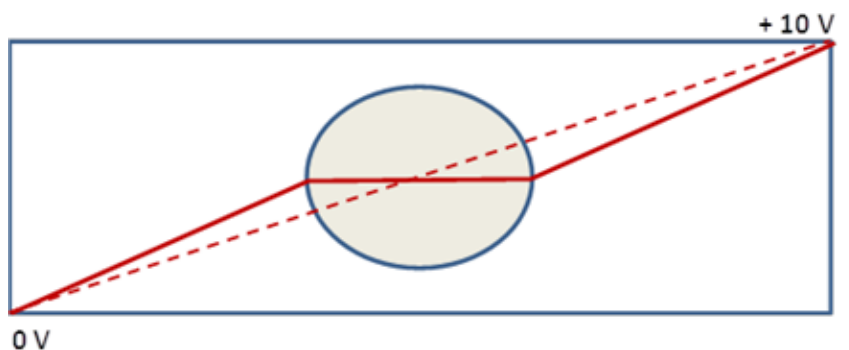

Fig. 8. Schematic of a high permittivity conducting rod in a low permittivity medium between two electrodes.

can be rationalized with knowledge of the basic physics of conductors.

In this scenario, we may expect to find the surface charge associated with the polarization, sketched in Fig. 8 and estimated in the exact analysis. Figure 9 shows a predicted surface charge density as a function of position around a circular inclusion.

Figure 10 shows the predicted Ohmic drop across a plain epoxy sample (dashed line). The dotted line gives a constant potential across the conductive inclusion.

In Fig. 11, the blue curve shows a plot of the measured surface potential against distance across a conductive sample in an epoxy matrix.

The measured values were obtained via the Scanning Kelvin Probe Microscopy (SKPM) mode on our Park Systems XE-100 Atomic Force Microscope (AFM) in air at normal room temperature and humidity. A standard SKPM conductive coated cantilever, NSC14-CrAu, was obtained from Park Systems. This cantilever had dimensions of $125 \mu \mathrm{m}$ for length, $35 \mu \mathrm{m}$ for width, a resonant frequency of $150 \mathrm{KHz}$, a quality factor of 233 , and tip radius of $50 \mathrm{~nm}$. During each measurement, a $4 \mathrm{~V}$ ac tip bias with a frequency of $18 \mathrm{KHz}$ was applied to the cantilever through a lock-in amplifier. Additionally a $10 \mathrm{~V}$ sample bias was applied through the sample via the AFM electrode clamp seen below

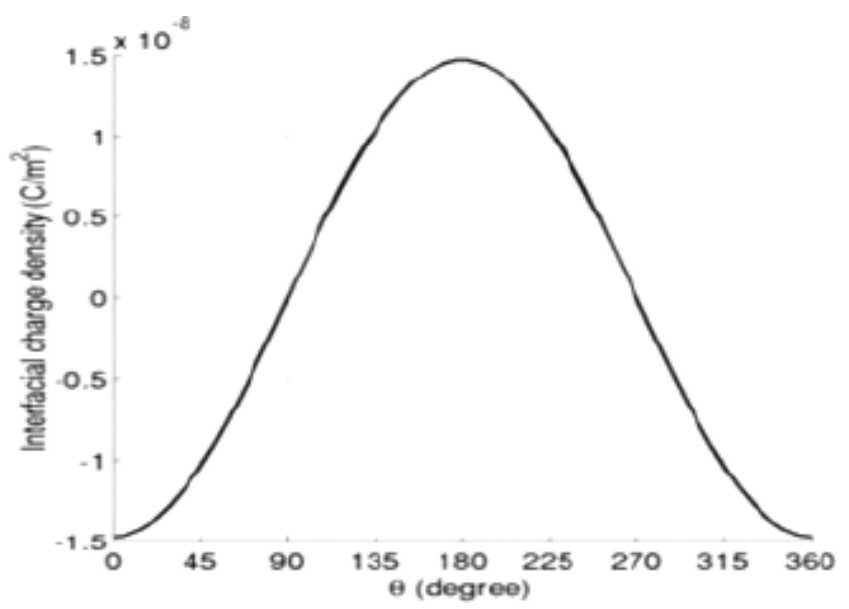

Fig. 9. Calculated interfacial charge around the periphery of the copper wire embedded in epoxy used in measurements. 


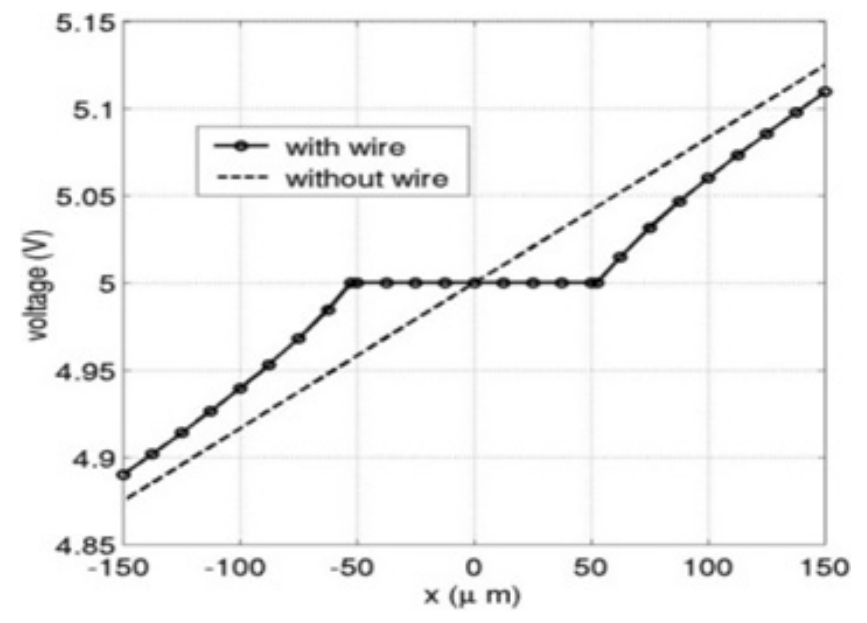

Fig. 10. Calculated potential distribution for experimental sample.

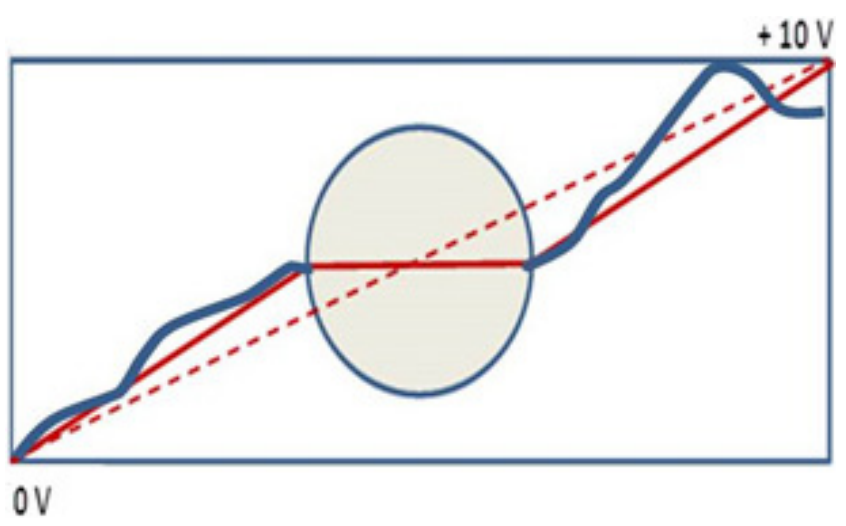

Fig. 11. (Color online) Measured values of potential across the physical sample.

in Fig. 13. Measurements were taken across the sample in a straight line near the source electrode all the way across the inclusion towards the ground electrode. The total distance traversed across the sample was $6 \mathrm{~mm}$.

The results confirm the calculated potential values seen in Fig. 10. The erratic behavior measured near the $10 \mathrm{~V}$ source electrode can be explained by considering the fringing effects of the electric field near the edge of the source electrode in contact with the specimen.

Figure 12 below shows the conductive circular inclusion with a diameter of $100 \mu \mathrm{m}$ embedded in an epoxy matrix.

By adapting our AFM to a method developed by Qi et al. ${ }^{24}$ for measuring, quantitatively, surface charge and local potential in samples via the noncontact Electrostatic Force Microscopy (EFM) mode, we quantitatively measured local sample surface potential (Fig. 12). Then based on the physical geometry of the AFM cantilever and sample, we calculated the local surface charge current. ${ }^{25}$

The experimental setup is illustrated below in Fig. 13. This clamping configuration provided a vector electric field parallel to the sample surface, with firm, even electrode contact over the sides of the specimen.

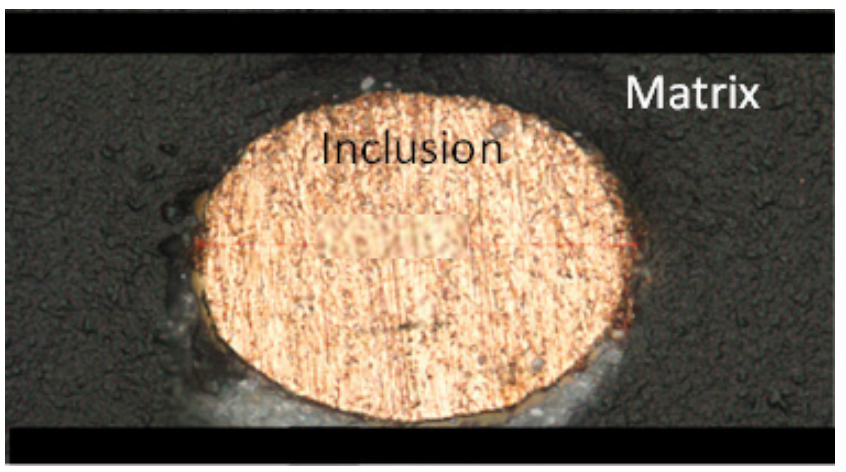

Fig. 12. Micrograph of copper rod in epoxy matrix.

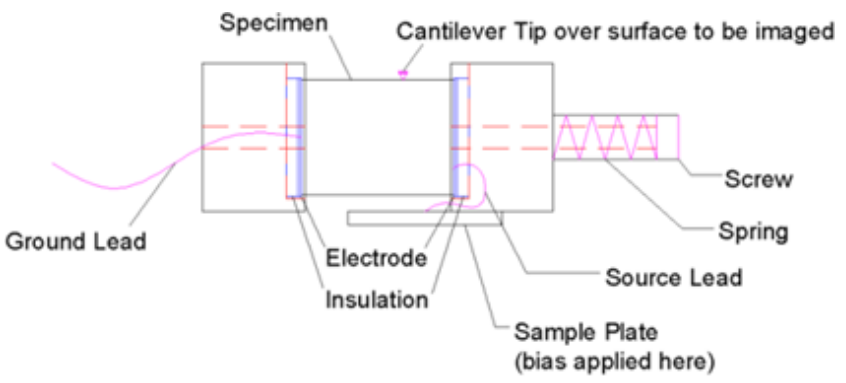

Fig. 13. Experimental configuration for measuring surface potential and interfacial charge distributions in an epoxy cube with an embedded copper wire.

The measurements were carried out using our Park Systems model XE-100 AFM utilizing the EFM internal mode with NSC14-CrAu cantilevers specified previously in this work. Figure 14 depicts the method. During an initial scan, the AFM recorded topographical information that was used to maintain the tip-sample distance precisely for the subsequent EFM pass over the sample. During the second scan, a precise stand-off distance was maintained, typically 50 to $100 \mathrm{~nm}$ so that the surface Van der Waals force did not influence the tip deflection. Therefore, any modulation of the tip vibration that caused a phase shift was associated with local charge stored at point locations on the surface. These shifts can be carefully calibrated with the help of a referential structure to yield quantitative measurements of local charge density.

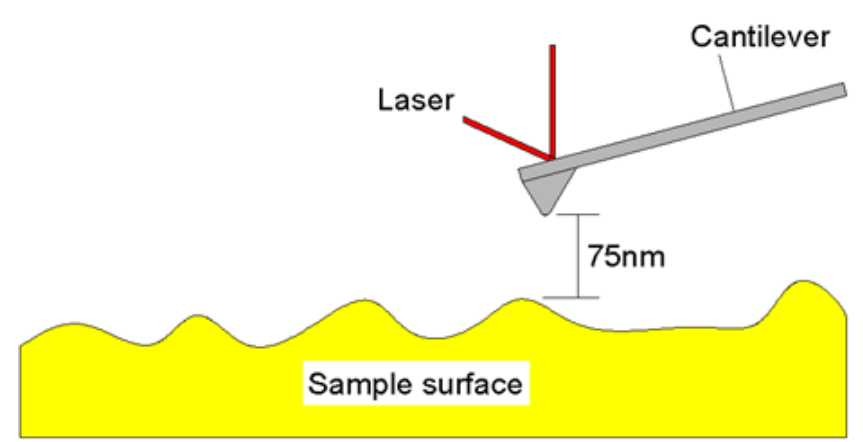

Fig. 14. AFM arrangement for measuring interfacial charge in a heterogeneous material. 


\section{Electrical Charge is Stored at the Interfaces in Heterogeneous Materials}

The total calculated interfacial charge density (free and bound charges) on the copper surface at the interface with the epoxy is distributed as shown in Fig. 9. The distribution varies around the periphery, with a maximum value of about $1.47 \times$ $10^{-8} \mathrm{C} / \mathrm{m}^{2}$. As we will see below, the experiments only measure the average charge in a finite region, so they cannot find a peak value at a point, as such. In order to examine the validity of our concepts and the limitations of our analysis, we have developed a methodology for experimental measurement of the local potential distributions and local charge distributions in heterogeneous materials.

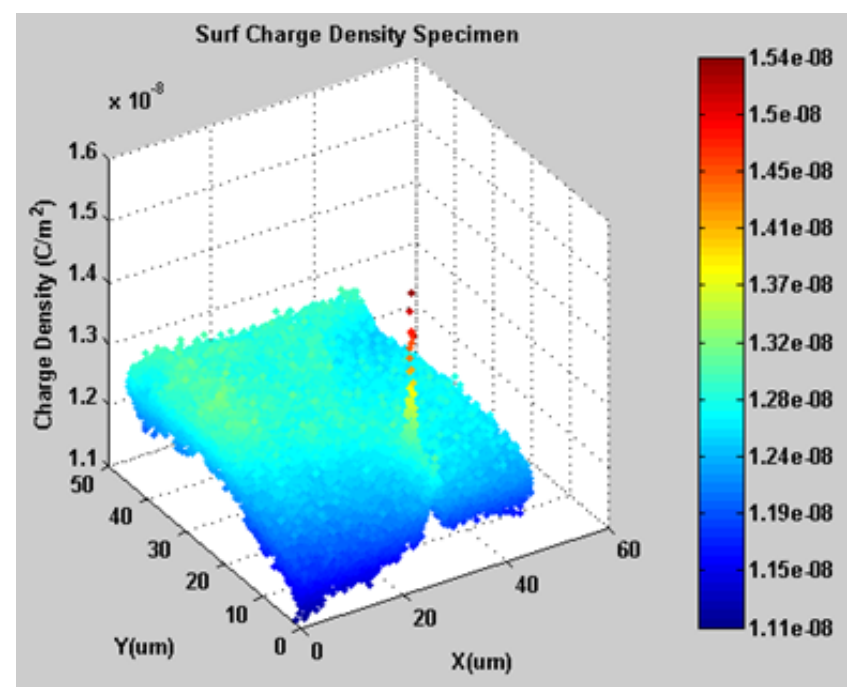

(a)

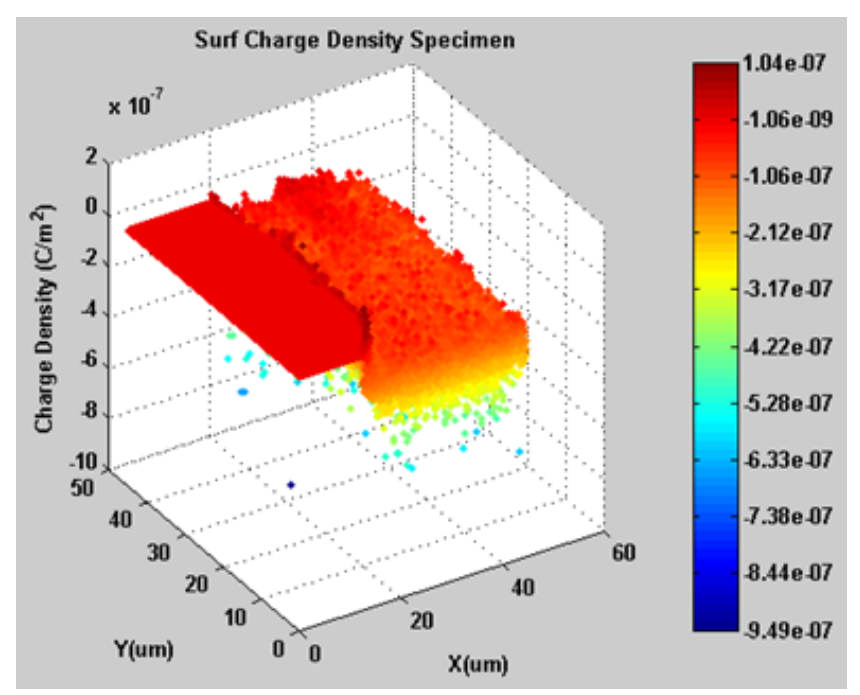

(b)

Fig. 15. (a) Measured surface charge density in the polarization regions on the left on the sample interface $\left(+1.2 \mathrm{e}-8 \mathrm{C} / \mathrm{m}^{2}\right)$, (b) right $\left(-9.66 \mathrm{e}-8 \mathrm{C} / \mathrm{m}^{2}\right)$ of the circular inclusion for an applied specimen bias voltage of $10 \mathrm{~V}$.
Measurements of the local charge distributions were successfully made; example data are shown in Fig. 15. It can be seen that the measured charge is somewhat distributed on either side of the (disordered) interface that is modeled as a planar surface in the analysis. This is expected, for several reasons. The singular change in the analytical slope of the voltage distribution that defines the surface charge is not singular in nature. Also, the interface between the high dielectric matrix and the high conductivity inclusion is not ordered in the material sense; the disorder occurs over a finite distance. However, the signs of the measured average charge in Fig. 15 match the signs of the polarization charges estimated in Fig. 11 and calculated precisely in Fig. 9. Moreover, the magnitudes for the surface charge measurements shown in Fig. 15, of the order of $1.3 \times 10^{-8} \mathrm{C} / \mathrm{m}^{2}$ compare well to the maximum calculated value of about $1.47 \times 10^{-8} \mathrm{C} / \mathrm{m}^{2}$. In addition, there are many other features in the experimental data that are similar to those predicted by our analysis.

\section{Conclusion}

We have presented the foundation for the rational design of the dielectric response of heterogeneous materials. Our design concepts are founded on the meso-scale details of constituent properties, shape, size, and interfaces to capture the emergent nature of the local constituent field interactions on the global response. At the local level, we find that the product of the electric displacement and the gradient of the potential (e.g., the electric field) controls the polarization and surface charge that collectively defines the global response. Extensive experimental validations confirm that large variations in the dielectric response can be designed and achieved for heterogeneous materials, exceeding the limits one might expect from any mixture estimate based on the constituent properties alone. There is an undeniable interaction which is not solely the summation of the individual set of material properties. Continuing work includes the extension of these concepts to include the dispersive nature of the dielectric behavior for which both frequencyand temperature-dependent local properties play a critical and substantial role. Additional work to systematically vary the inclusion position is underway, and will be enhanced by the fabrication of inserts with offsets between 2.3 and $0.5 \mathrm{~mm}$ off the center line of the major axis of the elliptical inclusion.

\section{Acknowledgments}

The authors gratefully acknowledge the support of the entirety of this work by the Energy Frontier Research Center for Heterogeneous Functional Materials, the HeteroFoaM Center, under DoE Grant no. DE-SC0001061 from the Office of Basic Energy Sciences. 


\section{References}

${ }^{1}$ M. Song, S. Cheng, H. Chen, W. Qin, K. Nam, S. Xu, X. Yang, A. Bongiorno, J. Lee, J. Bai, T. Tyson, J. Cho and M. Liu, Anomalous pseudocapacitive behavior of a nanostructured, Mixed-valent manganese oxide film for electrical energy storage, Nano Lett. 12, 3483 (2012).

${ }^{2}$ HeteroFoaM Center, Available at www.heterofoamcenter.com.

${ }^{3}$ P. Barber, S. Balasubramanian, Y. Anguchamy, S. Gong, A. Wibowo, H. Gao, H. J. Ploehn and H.-C. zur Loye, Polymer composite and nanocomposite dielectric materials for pulse power energy storage, Materials 2, 1697 (2009).

${ }^{4}$ A. C. Wibowo, M. D. Smith and H.-C. zur Loye, New 3D bismuth-oxo coordination polymers containing terephthalate-based ligands: Observation of $\mathrm{Bi} 2 \mathrm{O} 2$-Layer and $\mathrm{Bi} 4 \mathrm{O} 3$-chain motifs, Cryst. Eng. Comm. 13, 426 (2001).

${ }^{5}$ X. Liu, N. Wang, Z. Wu and W. Liu, Dielectric property of unidirectional triangle-shape carbon fiber reinforced polymeric composites, J. Compos. Mater. 48, 1143 (2013).

${ }^{6}$ K. L. Reifsnider, P. K. Majumdar and P. J. Fazzino, Material state changes as a basis for prognosis in aeronautical structures, $J$. Aeronaut. Soc. 113, 789 (2009).

${ }^{7}$ K. L. Reifsnider, W. K. Chiu, K. S. Brinkman, Y. Du, A. Nakaja, F. Rabbi and Q. Liu, Multiphysics design and development of heterogeneous functional materials for renewable energy devices: The HeteroFoaM story, J. Electrochem. Soc. 160, F470 (2013).

${ }^{8}$ K. S. Brinkman, E. B. Fox, P. Korinko, R. Lascola and F. Chen, Structure/property relations in bulk versus solution derived proton conducting ceramics of the form $\mathrm{SrCe} 0.95 \mathrm{Yb} 0.05 \mathrm{O} 3-\delta$ with applications in membrane separations, Proc. MRS (2013), doi: 10.1557/PROC-1256-N16-30.

${ }^{9}$ K. S. Brinkman, T. Iijima and H. Takamura, The oxygen permeation characteristics of $\mathrm{Bi} 1$ - xSrxFeO3 mixed ionic and electronic conducting ceramics, Solid State Ionics 181, 53 (2010).

${ }^{10}$ T.-W. Chou, Multifunctional carbon nanotube-based sensors for damage detection and self healing in structural composites, Delaware University, Newark, DE, AFOSR Final Report, Grant No: FA9550-09-1-0218, October 29, 2010.

${ }^{11}$ J. O. Aguilar, J. R. Bautista-Quijano and F. Aviles, Influence of carbon nanotube clustering on the electrical conductivity of polymer composite films, Express Polymer Lett. 4, 292 (2010).

${ }^{12}$ T. Joy and W. Strieder, Percolation in a thin ply of unidrectional composite, J. Compos. Mater. 13, 72 (1979).
${ }^{13}$ W. Lu, T.-W. Chou and E. T. Thostenson, A three-dimensional model of electrical percolations thresholds in carbon nanotubebased composites, Appl. Phys. Lett. 96, 1 (2010).

${ }^{14}$ B. K. P. Scaife, Principles of Dielectrics (Clarendon Press, Oxford, 1989).

${ }^{15}$ R. W. Sillars, The properties of a dielectric containing semiconducting particles, J. Inst. Electr. Eng. 80, 378 (1937).

${ }^{16}$ R. Pelster and U. Simon, Nanodispersions of conducting particles: Preparation, microstructure and dielectric properties, Colloid Polym. Sci. 277, 14 (1999).

${ }^{17}$ K. L. Reifsnider, F. Rabbi, J. Baker, J.-M. Adkins and G. Liu, Processing-property relationships in advanced multi-functional composite materials: Management of dielectric behavior, Proc. Int. Conf. Processing and Manufacturing of Advanced Materials, 2-6 December 2013.

${ }^{18}$ T. L. Bergman and N. Fekrazad, The effect of compressive load on proton exchange membrane fuel cell stack performance and behavior, ASME J. Heat Transfer 129, 1004 (2007).

${ }^{19}$ Q. Liu, Directly resoliving particles in an electric field: Local charge, force, torque, and applications, Int. J. Numer. Meth. Eng. 90, 537 (2012).

${ }^{20} \mathrm{Q}$. Liu, Physalis method for heterogeneous mixtures of dielectrics and conductors: Accurately simulating one millionparticles using a PC, J. Comput. Phys. 230, 8256 (2011).

${ }^{21}$ Q. Liu and K. L. Reifsnider, Heterogeneous mistures of elliptical particles: Directly resolving local and global properties and responses, J. Comput. Phys. 235, 161 (2013).

${ }^{22}$ S. B. Jones and S. P. Friedman, Particle shape effects on the effective permittivity of an isotropic or isotropic media consisting of aligned or randomly oriented ellipsoidal particles, Water Resources Res. 36, 2821 (2000).

${ }^{23}$ A. H. Sihvola and J. A. Kong, Effective permittivity of dielectric mixtures, IEEE Trans. Geosci. Remote Sens. 26, 420 (1988).

${ }^{24}$ G. Qi, Y. Yang, H. Yan, L. Guan, Y. Li, X. Qiu et al., Quantifying surface charge density by using an electric force microscope with a referential structure, J. Phys. Chem. C 113, 204 (2009).

${ }^{25}$ K. L. Reifsnider, Q. Liu, J.-M. Adkins, J. Baker, F. Rabbi and K. Brinkman, Relationship of micromorphology to charge storage and transfer properties in heterogeneous functioanl materials, Proc. World Conf. Computational Mechanics, 13-18 July 2012. 